\title{
ON COMPLETE METRIZABILITY OF THE HAUSDORFF METRIC TOPOLOGY
}

\author{
LÁSZLÓ ZSILINSZKY
}

(Communicated by Ken Ono)

\begin{abstract}
It is shown that there exists a nonseparable completely metrizable bounded metric space $(X, d)$ such that the hyperspace $C L(X)$ of the nonempty closed subsets of $X$ endowed with the Hausdorff metric $H_{d}$ is not completely metrizable.
\end{abstract}

\section{INTRODUCTION}

The main purpose of the paper is to demonstrate and clarify the differences between the hyperspace of a nonseparable versus a separable metric space regarding complete metrizability of the Hausdorff metric hypertopology. The Hausdorff metric topology $\tau_{H_{d}}$ on the hyperspace $C L(X)$ of nonempty closed subsets of a given metric space $(X, d)$ is one of the oldest and best-studied hypertopologies due to its applicability to various areas of mathematics [1,2, 4, 20. Various completenesstype properties of the Hausdorff metric topology are stock theorems in topology; e.g. $\left(C L(X), \tau_{H_{d}}\right)$ is compact (resp. totally bounded) iff $X$ is. More recently, local compactness 12 and cofinal completeness [6] have been characterized for $\left(C L(X), \tau_{H_{d}}\right)$, but a characterization of complete metrizability of $\left(C L(X), \tau_{H_{d}}\right)$ is unknown. There are partial results, however; indeed, the main reason for the interest in the Hausdorff metric is the following useful fact: if $(X, d)$ is a bounded complete metric space, then $\left(C L(X), H_{d}\right)$ is a complete metric space, where

$$
H_{d}\left(A_{0}, A_{1}\right)=\sup \left\{\left|d\left(x, A_{0}\right)-d\left(x, A_{1}\right)\right|: x \in X\right\} \text {, for } A_{0}, A_{1} \in C L(X),
$$

and $d(x, A)=\inf \{d(x, a): a \in A\}$ is the distance from $x \in X$ to $A \in C L(X)$. If $d$ is not bounded, $H_{d}$ is only an infinite-valued distance, which generates the topology $\tau_{H_{d}}$ on $C L(X)$. Moreover, $d^{\prime}=\min \{1, d\}$ is an equivalent to $d$, bounded metric on $X$ and $\tau_{H_{d^{\prime}}}=\tau_{H_{d}}$, so we get

Theorem 1.1. If $(X, d)$ is complete, then $\left(C L(X), \tau_{H_{d}}\right)$ is completely metrizable.

It is the purpose of this note to show that the previous theorem does not extend to a completely metrizable $(X, d)$, contrary to some claims in the literature [3]. In the main results, proved in Section 3, we use topological games - the so-called strong Choquet game and the Banach-Mazur game, reviewed in Section 2-to show that a nonseparable bounded completely metrizable metric space $(X, d)$ exists for which the corresponding Hausdorff metric topology is not completely metrizable. More

Received by the editors March 4, 2014 and, in revised form, July 11, 2016.

2010 Mathematics Subject Classification. Primary 54B20; Secondary 54E50, 54E52, 91A44.

Key words and phrases. Hausdorff distance, hyperspace, complete metrizability, strong Choquet game, Banach-Mazur game. 
generally, $\left(C L(X), \tau_{H_{d}}\right)$ lacks any closed-hereditary completeness property, since it contains a closed copy of the rationals; however, the hyperspace still contains a dense completely metrizable subspace, and thus is a Baire space.

To put this result in perspective, recall that it was Effros [16, Lemma] who showed that for $\left(C L(X), \tau_{H_{d}}\right)$ to be Polish (i.e. completely metrizable and separable), it is sufficient that $(X, d)$ is completely metrizable and totally bounded, which is in turn also necessary, since separability of $\left(C L(X), \tau_{H_{d}}\right)$ is equivalent to total boundedness of $X$ [4, Theorem 3.2.3.], and $X$ sits in $\left(C L(X), \tau_{H_{d}}\right)$ as a closed subspace. It is possible to improve on this result using the work of Costantini [10] about another related hyperspace topology, the so-called Wijsman topology $\tau_{W_{d}}$ : to explain, it is useful to view $C L(X)$ as sitting in the space $C(X)$ of real-valued continuous functions defined on $X$ via the identification $A \leftrightarrow d(\cdot, A)$, since, by (1.1), $\left(C L(X), \tau_{H_{d}}\right)$ is then a subspace of $C(X)$ with the uniform topology, while $\left(C L(X), \tau_{W_{d}}\right)$ is a subspace of $C(X)$ with the topology of pointwise convergence. This immediately implies that $\tau_{W_{d}} \subseteq \tau_{H_{d}}$, which yields

Theorem 1.2. If $(X, d)$ is Polish, then $\left(C L(X), \tau_{H_{d}}\right)$ is completely metrizable.

Proof. If $(\widetilde{X}, \widetilde{d})$ denotes the completion of $(X, d)$, it follows from [10] that the set $\left\{\operatorname{cl}_{\widetilde{X}}(A): A \in C L(X)\right\}$ is $G_{\delta}$ in $\left(C L(\widetilde{X}), \tau_{W_{\widetilde{d}}}\right)$; thus, it is $G_{\delta}$ in $\left(C L(\widetilde{X}), \tau_{H_{\widetilde{d}}}\right)$ as well. Therefore, by Theorem 1.1, $\left(C L(X), \tau_{H_{d}}\right)$ is completely metrizable, since mapping $A \in C L(X)$ onto the $\widetilde{X}$-closure of $A$ is an isometric embedding of $\left(C L(X), H_{d}\right)$ into $\left(C L(\widetilde{X}), H_{\widetilde{d}}\right)[16]$.

The Hausdorff distance is sensitive to its generating metric, because $\tau_{H_{d}}=\tau_{H_{d^{\prime}}}$ iff $d, d^{\prime}$ are uniformly equivalent metrics on $X$ [4, Theorem 3.3.2.]. Thus, it is not automatic to argue that complete metrizability of $(X, d)$ is sufficient for complete metrizability of $\left(C L(X), \tau_{H_{d}}\right)$ even though it is clearly necessary. Knowing that $\tau_{W_{d}}=\tau_{H_{d}}$ on $C L(X)$ iff $(X, d)$ is totally bounded [4, it is not surprising that the Hausdorff metric and Wijsman topologies interact in studying complete metrizability of the hyperspaces. However, when a totally bounded metric on $X$ is not available, i.e. when $X$ is not separable, the two topologies have no effect on each other, and so the wealth of completeness results on the Wijsman topology [5, 8, 11, 13, 27] is not applicable.

\section{Preliminaries}

Given a metric space $(X, d), A \in C L(X)$ and $\varepsilon>0$, denote by

$$
B_{d}(A, \varepsilon)=\{x \in X: d(x, A)<\varepsilon\}
$$

the open $\varepsilon$-hull of $A$, and use $B_{d}(x, \varepsilon)$ instead of $B_{d}(\{x\}, \varepsilon)$ for the open $\varepsilon$-ball about $x$. In addition to (1.1), there is an equivalent definition for the Hausdorff distance:

$$
H_{d}\left(A_{0}, A_{1}\right)=\inf \left\{\varepsilon>0: A_{0} \subseteq B_{d}\left(A_{1}, \varepsilon\right) \text { and } A_{1} \subseteq B_{d}\left(A_{0}, \varepsilon\right)\right\}
$$

whenever $A_{0}, A_{1} \in C L(X)$ [4, 17.

In the strong Choquet game $C h(Z), 19$ players $\alpha$ and $\beta$ take turns in choosing objects in the topological space $Z$ with an open base $\mathcal{B}: \beta$ starts by picking $\left(z_{0}, V_{0}\right)$ from $\mathcal{E}=\{(z, V) \in Z \times \mathcal{B}: z \in V\}$, and $\alpha$ responds by $U_{0} \in \mathcal{B}$ with $z_{0} \in U_{0} \subseteq V_{0}$. The next choice of $\beta$ is $\left(z_{1}, V_{1}\right) \in \mathcal{E}$ with $V_{1} \subseteq U_{0}$, and again $\alpha$ picks $U_{1}$ with $z_{1} \in U_{1} \subseteq V_{1}$, etc. Player $\alpha$ wins the run $\left(z_{0}, V_{0}\right), U_{0}, \ldots,\left(z_{n}, V_{n}\right), U_{n}, \ldots$ provided 
$\bigcap_{n} U_{n}=\bigcap_{n} V_{n} \neq \emptyset$; otherwise, $\beta$ wins. A strategy in $C h(Z)$ for $\alpha$ (resp. $\beta$ ) is a function $\sigma: \mathcal{E}^{<\omega} \rightarrow \mathcal{B}$ (resp. $\sigma: \mathcal{B}^{<\omega} \rightarrow \mathcal{E}$ ) such that

$$
\begin{gathered}
z_{n} \in \sigma\left(\left(z_{0}, V_{0}\right), \ldots,\left(z_{n}, V_{n}\right)\right) \subseteq V_{n} \text { for all }\left(\left(z_{0}, V_{0}\right), \ldots,\left(z_{n}, V_{n}\right)\right) \in \mathcal{E}^{<\omega} \\
\left(\text { resp. } \sigma(\emptyset)=\left(z_{0}, V_{0}\right) \text { and } V_{n} \subseteq U_{n-1}, \text { where } \sigma\left(U_{0}, \ldots, U_{n-1}\right)=\left(z_{n}, V_{n}\right)\right. \\
\text { for all } \left.\left(U_{0}, \ldots, U_{n-1}\right) \in \mathcal{B}^{n}, n \geq 1\right) .
\end{gathered}
$$

A strategy $\sigma$ for $\alpha$ (resp. $\beta$ ) is a winning strategy if $\alpha$ (resp. $\beta$ ) wins every run of $C h(Z)$ compatible with $\sigma$, i.e. such that $\left.\sigma\left(z_{0}, V_{0}\right), \ldots,\left(z_{n}, V_{n}\right)\right)=U_{n}$ for all $n<\omega$ (resp. $\sigma(\emptyset)=\left(z_{0}, V_{0}\right)$ and $\sigma\left(U_{0}, \ldots, U_{n-1}\right)=\left(z_{n}, V_{n}\right)$ for all $\left.n \geq 1\right)$. The strong Choquet game $C h(Z)$ is $\alpha$-, $\beta$-favorable, respectively, provided $\alpha$, resp. $\beta$, has a winning strategy in $C h(Z)$. This game has been studied in general topological spaces [7, 14, 15, 22, 28]; however, the two fundamental results about it concern metrizable ones:

- Choquet [9, 19]. A metrizable space $X$ is completely metrizable if and only if $C h(X)$ is $\alpha$-favorable.

- Debs-Porada-Telgársky [14,24,25]. A metrizable space $X$ contains a closed copy of the rationals if and only if $C h(X)$ is $\beta$-favorable.

The Banach-Mazur game $B M(Z)$ (see [18, also referred to as the Choquet game [19]) is played as the strong Choquet game, except $\beta$ 's choice is only a nonempty open set contained in the previous choice of $\alpha$. The notions of $\alpha-\beta$-favorability of $B M(Z)$ are defined analogously to those of $C h(Z)$. Two key results about the Banach-Mazur game are as follows:

- Oxtoby [23,26]. A metrizable space $X$ contains a dense completely metrizable subspace if and only if $B M(X)$ is $\alpha$-favorable.

- Oxtoby-Krom [18, 19, 23]. A topological space $X$ is a Baire space (i.e. countable intersections of dense open subsets of $X$ are dense) if and only if $B M(X)$ is not $\beta$-favorable.

\section{MAIN RESUltS}

Our main result is as follows:

Theorem 3.1. There exists a nonseparable bounded metric space $(X, d)$ such that

(1) $X$ is completely metrizable;

(2) $\left(C L(X), H_{d}\right)$ contains a closed copy of the rationals; in particular, $\left(C L(X), H_{d}\right)$ is not completely metrizable;

(3) $\left(C L(X), H_{d}\right)$ is $\alpha$-favorable in the Banach-Mazur game; in particular, $\left(C L(X), H_{d}\right)$ is a Baire space.

Proof. (1) Consider the product space $\mathbb{R}^{\omega}$, where $\mathbb{R}$ has the discrete topology. This topology is metrizable by the Baire metric [17. Example 4.2.12(6)]

$$
d(x, y)=\frac{1}{\min \{n+1: x(n) \neq y(n)\}}
$$

for $x, y \in \mathbb{R}^{\omega}$. Denote

$$
F=\left\{x \in \mathbb{R}^{\omega}: x(0) \neq 0 \text { and } x(k)=0 \text { for all } k>0\right\},
$$

and put $X=\mathbb{R}^{\omega} \backslash F$. It is clear that $F$ is closed in $\mathbb{R}^{\omega}$, so $X$ is an open subspace of the complete space $\left(\mathbb{R}^{\omega}, d\right)$, and hence $(X, d)$ is completely metrizable. 
(2) By the Debs-Porada-Telgársky Theorem, we need to show that $\left(C L(X), H_{d}\right)$ is $\beta$-favorable in the strong Choquet game: let $\left\{I_{n}^{0} \subset \mathbb{R} \backslash\{0\}: n<\omega\right\}$ be a sequence of pairwise disjoint closed bounded intervals, and denote by $I_{0}$ their union. For each $t \in I_{0}$ define $x_{t}^{0} \in X$ via

$$
x_{t}^{0}(k)= \begin{cases}t, & \text { if } t \in I_{n}^{0}, k=0 \text { or } k>n+1, \\ 0, & \text { if } t \in I_{n}^{0}, 1 \leq k \leq n+1 .\end{cases}
$$

Define $A_{0}=\left\{x_{t}^{0}: t \in I_{0}\right\} \in C L(X), \mathbf{V}_{0}=B_{H_{d}}\left(A_{0}, 1\right)$, and let $\left(A_{0}, \mathbf{V}_{0}\right)$ be $\beta$ 's initial step in $C h\left(C L(X), H_{d}\right)$. Assume that $\mathbf{U}_{0}=B_{H_{d}}\left(A_{0}, \frac{1}{n_{0}}\right)$ is $\alpha$ 's response, where $1 \leq n_{0}<\omega$.

Let $\left\{I_{n}^{1} \subset I_{n_{0}+1}^{0}: n<\omega\right\}$ be a sequence of pairwise disjoint closed bounded intervals with union $I_{1}$. For each $t \in I_{0} \backslash I_{1}$ define $x_{t}^{1}=x_{t}^{0}$, and for $t \in I_{1}$ put

$$
x_{t}^{1}(k)= \begin{cases}x_{t}^{0}(k), & \text { if } t \in I_{n}^{1}, k \leq n_{0}, \\ 0, & \text { if } t \in I_{n}^{1}, n_{0}<k \leq n_{0}+n+1, \\ t, & \text { if } t \in I_{n}^{1}, k>n_{0}+n+1 .\end{cases}
$$

Define $A_{1}=\left\{x_{t}^{1}: t \in I_{0}\right\} \in C L(X)$ and $\mathbf{V}_{1}=B_{H_{d}}\left(A_{1}, \frac{1}{1+n_{0}}\right)$, and let $\left(A_{1}, \mathbf{V}_{1}\right)$ be $\beta$ 's next step in $C h\left(C L(X), H_{d}\right)$.

Assume we have defined a partial run $\left(A_{0}, \mathbf{V}_{0}\right), \mathbf{U}_{0}, \ldots,\left(A_{m}, \mathbf{V}_{m}\right), \mathbf{U}_{m}$ of the strong Choquet game in $\left(C L(X), H_{d}\right)$ for some $m<\omega$, where

$$
\mathbf{U}_{i}=B_{H_{d}}\left(A_{i}, \frac{1}{\sum_{j \leq i} n_{j}}\right)
$$

for some $1 \leq n_{i}<\omega$ whenever $i \leq m$. Moreover, for each $1 \leq i \leq m$ a sequence $\left\{I_{n}^{i} \subset I_{n_{i-1}+1}^{i-1}: n<\omega\right\}$ of pairwise disjoint closed bounded intervals with union $I_{i}$ is chosen, as well as $x_{t}^{i} \in X$ for each $t \in I_{0}$ so that $x_{t}^{i}=x_{t}^{i-1}$ whenever $t \in I_{0} \backslash I_{i}$, and for $t \in I_{i}$,

$$
x_{t}^{i}(k)= \begin{cases}x_{t}^{i-1}(k), & \text { if } t \in I_{n}^{i}, k \leq \sum_{j<i} n_{j}, \\ 0, & \text { if } t \in I_{n}^{i}, \sum_{j<i} n_{j}<k \leq 1+n+\sum_{j<i} n_{j}, \\ t, & \text { if } t \in I_{n}^{i}, k>1+n+\sum_{j<i} n_{j} .\end{cases}
$$

Then $A_{i}=\left\{x_{t}^{i}: t \in I_{0}\right\}$ and $\mathbf{V}_{i}=B_{H_{d}}\left(A_{i}, \frac{1}{1+\sum_{j<i} n_{j}}\right)$.

Choose a sequence of pairwise disjoint closed bounded intervals $\left\{I_{n}^{m+1} \subset I_{n_{m}+1}^{m}\right.$ : $n<\omega\}$ with union $I_{m+1}$, define $x_{t}^{m+1}=x_{t}^{m}$ for each $t \in I_{0} \backslash I_{m+1}$, and for $t \in I_{m+1}$ put

$$
x_{t}^{m+1}(k)= \begin{cases}x_{t}^{m}(k), & \text { if } t \in I_{n}^{m+1}, k \leq \sum_{i \leq m} n_{i}, \\ 0, & \text { if } t \in I_{n}^{m+1}, \sum_{i \leq m} n_{i}<k \leq 1+n+\sum_{i \leq m} n_{i}, \\ t, & \text { if } t \in I_{n}^{m+1}, k>1+n+\sum_{i \leq m} n_{i} .\end{cases}
$$

Define $A_{m+1}=\left\{x_{t}^{m+1}: t \in I_{0}\right\}$ and $\mathbf{V}_{m+1}=B_{H_{d}}\left(A_{m+1}, \frac{1}{1+\sum_{i \leq m} n_{i}}\right)$.

Claim 3.1.1. $\mathbf{V}_{m+1} \subseteq \mathbf{U}_{m}$. 
Indeed, if $A \in \mathbf{V}_{m+1}$, then $A \subseteq B_{d}\left(A_{m+1}, \frac{1}{1+\sum_{i<m} n_{i}}\right)$, so for all $a \in A$ there is some $x_{t}^{m+1} \in A_{m+1}$ with $d\left(a, x_{t}^{m+1}\right)<\frac{1}{1+\sum_{i \leq m} n_{i}}$, which implies that

$$
a(k)=x_{t}^{m+1}(k) \text { for all } k \leq \sum_{i \leq m} n_{i} .
$$

If $t \in I_{0} \backslash I_{m+1}$, then

$$
d\left(a, x_{t}^{m}\right)=d\left(a, x_{t}^{m+1}\right)<\frac{1}{1+\sum_{i \leq m} n_{i}}<\frac{1}{\sum_{i \leq m} n_{i}} ;
$$

if $t \in I_{m+1}$, then $t \in I_{n}^{m+1}$ for some $n<\omega$. It follows from the definition of $x_{t}^{m+1}$ and (3.1) that

$$
d\left(a, x_{t}^{m}\right) \leq \frac{1}{1+\sum_{i \leq m} n_{i}}<\frac{1}{\sum_{i \leq m} n_{i}},
$$

so we have $A \subseteq B_{d}\left(A_{m}, \frac{1}{\sum_{i \leq m} n_{i}}\right)$. A similar argument shows that

$$
A_{m+1} \subseteq B_{d}\left(A, \frac{1}{1+\sum_{i \leq m} n_{i}}\right) \text { implies that } A_{m} \subseteq B_{d}\left(A, \frac{1}{\sum_{i \leq m} n_{i}}\right),
$$

thus $A \in \mathbf{U}_{m}$. As a consequence of Claim 3.1.1, we have that putting

$$
\sigma_{C h}(\emptyset)=\left(A_{0}, \mathbf{V}_{0}\right) \text { and } \sigma_{C h}\left(\mathbf{U}_{0}, \ldots, \mathbf{U}_{m}\right)=\left(A_{m+1}, \mathbf{V}_{m+1}\right) \text { whenever } m<\omega
$$

defines a strategy for player $\beta$ in the strong Choquet game on $\left(C L(X), H_{d}\right)$. We will be done if we prove

Claim 3.1.2. $\sigma_{C h}$ is a winning strategy for $\beta$ in $C h\left(C L(X), H_{d}\right)$.

To show this, consider a run

$$
\left(A_{0}, \mathbf{V}_{0}\right), \mathbf{U}_{0}, \ldots,\left(A_{m}, \mathbf{V}_{m}\right), \mathbf{U}_{m}, \ldots
$$

of $C h\left(C L(X), H_{d}\right)$ compatible with $\sigma_{C h}$, and assume $A \in \bigcap_{m<\omega} \mathbf{V}_{m}$. If we choose some $t \in \bigcap_{m<\omega} I_{n_{m}+1}^{m}$, note that for every $m<\omega$,

$$
x_{t}^{m}(k)=0 \text { for all } 0<k \leq 1+\sum_{i \leq m} n_{i} .
$$

Since $A \in \mathbf{V}_{0}$, there is some $a \in A$ with $d\left(x_{t}^{0}, a\right)<1$, thus

$$
a(0)=x_{t}^{0}(0)=t .
$$

Since $a \in X$, there exists $0<k$ so that

$$
a(k) \neq 0 .
$$

Choose $m<\omega$ so that $k \leq 1+\sum_{i \leq m} n_{i}$. Since $A \in \mathbf{V}_{m}$, there exists an $x_{t^{\prime}}^{m} \in A_{m}$ with $d\left(a, x_{t^{\prime}}^{m}\right)<\frac{1}{1+\sum_{i \leq m} n_{i}}$, which implies that

$$
\begin{gathered}
x_{t^{\prime}}^{m}(0)=a(0) \text { and } \\
x_{t^{\prime}}^{m}(k)=a(k) .
\end{gathered}
$$


Using (3.3), (3.5) we get

$$
t^{\prime}=x_{t^{\prime}}^{m}(0)=a(0)=x_{t}^{0}(0)=t,
$$

so $t^{\prime}=t$. This would yield, by (3.6), (3.2), that

$$
a(k)=x_{t^{\prime}}^{m}(k)=x_{t}^{m}(k)=0,
$$

which contradicts (3.4). In conclusion, $\bigcap_{m<\omega} \mathbf{V}_{m}=\emptyset$, which means that $\beta$ wins.

(3) Let $\mathbf{V}_{0}$ be $\beta$ 's initial step in $B M\left(C L(X), H_{d}\right)$, where $\mathbf{V}_{0}=B_{H_{d}}\left(A_{0}, \frac{1}{n_{0}}\right)$ for some $A_{0} \in C L(X)$ and $n_{0} \geq 1$. For each $a_{0} \in A_{0}$ define $x_{a_{0}} \in X$ via

$$
x_{a_{0}}(k)= \begin{cases}a_{0}(k), & \text { if } k<2 n_{0}-1, \\ n_{0}+1, & \text { if } k \geq 2 n_{0}-1,\end{cases}
$$

and put $C_{0}=\left\{x_{a_{0}}: a_{0} \in A_{0}\right\}$. Then $C_{0} \in C L(X)$, and $H_{d}\left(A_{0}, C_{0}\right) \leq \frac{1}{2 n_{0}}$. Define $\mathbf{U}_{0}=B_{H_{d}}\left(C_{0}, \frac{1}{2 n_{0}}\right)$. Then $\mathbf{U}_{0} \subseteq \mathbf{V}_{0}$ (since if $A \in \mathbf{U}_{0}$, then $H_{d}\left(A, C_{0}\right)<\frac{1}{2 n_{0}}$, so $\left.H_{d}\left(A_{0}, A\right) \leq H_{d}\left(A, C_{0}\right)+H_{d}\left(C_{0}, A_{0}\right)<\frac{1}{n_{0}}\right)$, so we can take $\mathbf{U}_{0}$ as $\alpha$ 's first step in $B M\left(C L(X), H_{d}\right)$. Let $\mathbf{V}_{1}=B_{H_{d}}\left(A_{1}, \frac{1}{n_{1}}\right) \subseteq \mathbf{U}_{0}$ be $\beta$ 's next step, where $A_{1} \in C L(X)$ and $n_{1} \geq 2 n_{0}$. Then $H_{d}\left(A_{1}, C_{0}\right)<\frac{1}{2 n_{0}}$, so for all $a_{0} \in A_{0}$ there exists $a_{1} \in A_{1}$ such that $d\left(a_{1}, x_{a_{0}}\right)<\frac{1}{2 n_{0}}$, thus

$$
a_{1}(k)= \begin{cases}a_{0}(k), & \text { if } k<2 n_{0}-1, \\ n_{0}+1, & \text { if } k=2 n_{0}-1 .\end{cases}
$$

For each $a_{1} \in A_{1}$ define $x_{a_{1}} \in X$ via

$$
x_{a_{1}}(k)= \begin{cases}a_{1}(k), & \text { if } k<2 n_{1}-1, \\ 1+n_{0}+n_{1}, & \text { if } k \geq 2 n_{1}-1,\end{cases}
$$

and put $C_{0}=\left\{x_{a_{1}}: a_{1} \in A_{1}\right\}$. Then $C_{1} \in C L(X)$, and $H_{d}\left(A_{1}, C_{1}\right) \leq \frac{1}{2 n_{1}}$, so $\mathbf{U}_{1}=B_{H_{d}}\left(C_{1}, \frac{1}{2 n_{1}}\right) \subseteq \mathbf{V}_{1}$.

Assume we have defined a partial run $\mathbf{V}_{0}, \mathbf{U}_{0}, \ldots, \mathbf{V}_{m}, \mathbf{U}_{m}$ of the Banach-Mazur game in $\left(C L(X), H_{d}\right)$, where

$$
\mathbf{V}_{i}=B_{H_{d}}\left(A_{i}, \frac{1}{n_{i}}\right) \text { and } \mathbf{U}_{i}=B_{H_{d}}\left(C_{i}, \frac{1}{2 n_{i}}\right)
$$

for some $2 n_{i-1} \leq n_{i}<\omega$ whenever $i \leq m$ (for convenience, define $n_{-1}=\frac{1}{2}$ ). Moreover, for each $i \leq m$ let $C_{i}=\left\{x_{a_{i}}: a_{i} \in A_{i}\right\}$, where

$$
x_{a_{i}}(k)= \begin{cases}a_{i}(k), & \text { if } k<2 n_{i}-1, \\ 1+\sum_{j \leq i} n_{j}, & \text { if } k \geq 2 n_{i}-1 .\end{cases}
$$

Take $\mathbf{V}_{m+1}=B_{H_{d}}\left(A_{m+1}, \frac{1}{n_{m+1}}\right) \subseteq \mathbf{U}_{m}$. For any $a_{m+1} \in A_{m+1}$ define

$$
y_{a_{m+1}}(k)= \begin{cases}a_{m+1}(k), & \text { if } k<n_{m+1}, \\ 2+\sum_{i \leq m} n_{i}, & \text { if } k \geq n_{m+1} .\end{cases}
$$


Then $\left\{y_{a_{m+1}}: a_{m+1} \in A_{m+1}\right\} \in \mathbf{V}_{m+1} \subseteq \mathbf{U}_{m}$, so there exists $x_{a_{m}} \in C_{m}$ for some $a_{m} \in A_{m}$ so that $d\left(y_{a_{m+1}}, x_{a_{m}}\right)<\frac{1}{2 n_{m}}$. If $n_{m+1}<2 n_{m}$, then

$$
\begin{gathered}
y_{a_{m+1}}\left(2 n_{m}-1\right)=2+\sum_{i \leq m} n_{i} \text { and } \\
x_{a_{m}}\left(2 n_{m}-1\right)=1+\sum_{i \leq m} n_{i},
\end{gathered}
$$

so $d\left(y_{a_{m+1}}, x_{a_{m}}\right) \geq \frac{1}{2 n_{m}}$, which is impossible, thus $n_{m+1} \geq 2 n_{m}$. It also follows from $\mathbf{V}_{m+1} \subseteq \mathbf{U}_{m}$ that $H_{d}\left(A_{m+1}, C_{m}\right)<\frac{1}{2 n_{m}}$. Hence, for each $a_{m} \in A_{m}$ there exists $a_{m+1} \in A_{m+1}$ with $d\left(x_{a_{m}}, a_{m+1}\right)<\frac{1}{2 n_{m}}$, so

$$
a_{m+1}(k)= \begin{cases}a_{m}(k), & \text { if } k<2 n_{m}-1, \\ 1+\sum_{i \leq m} n_{i}, & \text { if } k=2 n_{m}-1 .\end{cases}
$$

Define $C_{m+1}=\left\{x_{a_{m+1}}: a_{m+1} \in A_{m+1}\right\}$, where

$$
x_{a_{m+1}}(k)= \begin{cases}a_{m+1}(k), & \text { if } k<2 n_{m+1}-1, \\ 1+\sum_{i \leq m+1} n_{i}, & \text { if } k \geq 2 n_{m+1}-1,\end{cases}
$$

and put $\mathbf{U}_{m+1}=B_{H_{d}}\left(C_{m+1}, \frac{1}{2 n_{m+1}}\right)$. Note that $H_{d}\left(A_{m+1}, C_{m+1}\right) \leq \frac{1}{2 n_{m+1}}$, so $\mathbf{U}_{m+1} \subseteq \mathbf{V}_{m+1}$, since if $A \in \mathbf{U}_{m+1}$, then $H_{d}\left(C_{m+1}, A\right)<\frac{1}{2 n_{m+1}}$, thus, $H_{d}\left(A_{m+1}, A\right)$ $\leq H_{d}\left(A_{m+1}, C_{m+1}\right)+H_{d}\left(C_{m+1}, A\right)<\frac{1}{n_{m+1}}$. This means that putting

$$
\sigma_{B M}\left(\mathbf{V}_{0}, \ldots, \mathbf{V}_{m}\right)=\mathbf{U}_{m} \text { for all } m<\omega
$$

defines a strategy for $\alpha$ in $B M\left(C L(X), H_{d}\right)$.

Claim 3.1.3. $\sigma_{B M}$ is a winning strategy for $\alpha$ in $B M\left(C L(X), H_{d}\right)$.

To show this, consider a run $\mathbf{V}_{0}, \mathbf{U}_{0}, \ldots, \mathbf{V}_{m}, \mathbf{U}_{m}, \ldots$ of the Banach-Mazur game in $\left(C L(X), H_{d}\right)$ compatible with $\sigma_{B M}$. For any $m<\omega$ and $a_{m} \in A_{m}$ we get an $a_{m+1} \in A_{m+1}$ satisfying (3.9). For any $a_{0} \in A_{0}$, define

$$
A_{1}\left[a_{0}\right]=\left\{a_{1} \in A_{1}: a_{1}(k)=a_{0}(k) \text { for all } k<2 n_{0}-1\right\},
$$

which is nonempty by (3.7). Assume by induction that we have defined $A_{m}\left[a_{m-1}\right] \neq$ $\emptyset$ for some $a_{m-1} \in A_{m-1}$ and $m \geq 1$. For every $a_{m} \in A_{m}\left[a_{m-1}\right]$ put

$$
A_{m+1}\left[a_{m}\right]=\left\{a_{m+1} \in A_{m+1}: a_{m+1}(k)=a_{m}(k) \text { for all } k<2 n_{m}-1\right\},
$$

which is nonempty by (3.9); for convenience, also define $A_{0}\left[a_{-1}\right]=A_{0}$. Denote

$$
P=\left\{\left(a_{m}\right)_{m \geq 0}: a_{m} \in A_{m}\left[a_{m-1}\right] \text { for all } m \geq 0\right\},
$$

and for any $p=\left(a_{m}\right)_{m \geq 0} \in P$ define $s_{p}$ as follows:

$$
s_{p}(k)= \begin{cases}a_{0}(k), & \text { if } k<2 n_{0}-1, \\ a_{m}(k), & \text { if } 2 n_{m-1}-1 \leq k<2 n_{m}-1, m \geq 1 .\end{cases}
$$

Note, by (3.9), that $s_{p}\left(2 n_{m}-1\right)=1+\sum_{i \leq m} n_{i}$ for every $m<\omega$, so $s_{p} \in X$ for each $p \in P$. Denote by $S$ the $X$-closure of the set $\left\{s_{p}: p \in P\right\}$.

Given any $s_{p} \in S$, we have a sequence $p=\left(a_{m}\right)_{m \geq 0} \in P$ such that $a_{i}(k)=$ $a_{i-1}(k)$ for all $1 \leq i \leq m$ and $k<2 n_{i}-1$, which implies by (3.11) that $a_{m}(k)=$ $s_{p}(k)$ for all $k<2 n_{m}-1$. 
It follows that $d\left(s_{p}, A_{m}\right) \leq d\left(s_{p}, a_{m}\right) \leq \frac{1}{2 n_{m}}$, so $d\left(s, A_{m}\right) \leq \frac{1}{2 n_{m}}<\frac{1}{n_{m}}$ for each $s \in S$, thus

$$
S \subseteq B_{H_{d}}\left(A_{m}, \frac{1}{n_{m}}\right)
$$

Furthermore, for each $1 \leq i \leq m, A_{i} \in \mathbf{V}_{i} \subseteq \mathbf{U}_{i-1}$, so for any $a_{i} \in A_{i}$ there exists $a_{i-1} \in A_{i-1}$ with $d\left(x_{a_{i-1}}, a_{i}\right)<\frac{1}{2 n_{i-1}}$, which means that $a_{i}(k)=x_{a_{i-1}}(k)$ for each $k \leq 2 n_{i-1}-1$, so by (3.8),

$$
a_{i}(k)=a_{i-1}(k) \text { for each } k<2 n_{i-1}-1 \text {; }
$$

moreover, if $i>m$ we can choose, by (3.9), $a_{i} \in A_{i}$ so that (3.13) is satisfied. It follows that $a_{i} \in A_{i}\left[a_{i-1}\right]$ for all $1 \leq i$; thus $p=\left(a_{i}\right)_{i \geq 0} \in P$ and $s_{p}(k)=a_{m}(k)$ for all $k<2 n_{m}-1$.

This implies that $d\left(a_{m}, S\right) \leq d\left(a_{m}, s_{p}\right) \leq \frac{1}{2 n_{m}}<\frac{1}{n_{m}}$, so

$$
A_{m} \subseteq B_{H_{d}}\left(S, \frac{1}{n_{m}}\right) .
$$

In conclusion, by (3.12), (3.14) we have that $H_{d}\left(A_{m}, S\right)<\frac{1}{n_{m}}$; thus $S \in \mathbf{V}_{m}$, which implies that $S \in \bigcap_{m<\omega} \mathbf{V}_{m}$, and so $\alpha$ wins.

Corollary 3.2. There exists a completely metrizable bounded metric space $X$ with compatible metrics $d, d^{\prime}$ so that $C h\left(C L(X), H_{d}\right)$ is $\alpha$-favorable and $C h\left(C L(X), H_{d^{\prime}}\right)$ is $\beta$-favorable.

\section{REFERENCES}

[1] Jean-Pierre Aubin, Applied abstract analysis, Exercises by Bernard Cornet and Hervé Moulin, translated from the French by Carole Labrousse, Pure and Applied Mathematics, WileyInterscience [John Wiley \& Sons], New York-London-Sydney, 1977. MR470034

[2] C. Castaing and M. Valadier, Convex analysis and measurable multifunctions, Lecture Notes in Mathematics, Vol. 580, Springer-Verlag, Berlin-New York, 1977. MR0467310

[3] T. Banakh and R. Voytsitskyy, Characterizing metric spaces whose hyperspaces are homeomorphic to $l_{2}$, Colloq. Math. 113 (2008), no. 2, 223-229, DOI 10.4064/cm113-2-4. MR2425083

[4] Gerald Beer, Topologies on closed and closed convex sets, Mathematics and its Applications, vol. 268, Kluwer Academic Publishers Group, Dordrecht, 1993. MR.1269778

[5] Gerald Beer, A Polish topology for the closed subsets of a Polish space, Proc. Amer. Math. Soc. 113 (1991), no. 4, 1123-1133, DOI 10.2307/2048792. MR.1065940

[6] Gerald Beer and Giuseppe Di Maio, Confinal completeness of the Hausdorff metric topology, Fund. Math. 208 (2010), no. 1, 75-85, DOI 10.4064/fm208-1-5. MR.2609221

[7] H. R. Bennett, D. J. Lutzer, and G. M. Reed, Domain representability and the Choquet game in Moore and BCO-spaces, Topology Appl. 155 (2008), no. 5, 445-458, DOI 10.1016/j.topol.2007.10.005. MR.2380929

[8] J. Chaber and R. Pol, Note on the Wijsman hyperspaces of completely metrizable spaces (English, with English and Italian summaries), Boll. Unione Mat. Ital. Sez. B Artic. Ric. Mat. (8) 5 (2002), no. 3, 827-832. MR1934383

[9] Gustave Choquet, Lectures on analysis. Vol. I: Integration and topological vector spaces, Edited by J. Marsden, T. Lance and S. Gelbart, W. A. Benjamin, Inc., New York-Amsterdam, 1969. MR0250011

[10] C. Costantini, Every Wijsman topology relative to a Polish space is Polish, Proc. Amer. Math. Soc. 123 (1995), no. 8, 2569-2574, DOI 10.2307/2161290. MR.1273484

[11] Camillo Costantini, On the hyperspace of a non-separable metric space, Proc. Amer. Math. Soc. 126 (1998), no. 11, 3393-3396, DOI 10.1090/S0002-9939-98-04956-9. MR.1618729 
[12] Camillo Costantini, Sandro Levi, and Jan Pelant, Compactness and local compactness in hyperspaces, Topology Appl. 123 (2002), no. 3, 573-608, DOI 10.1016/S0166-8641(01)00222X. MR.1924053

[13] Jiling Cao and A. H. Tomita, The Wijsman hyperspace of a metric hereditarily Baire space is Baire, Topology Appl. 157 (2010), no. 1, 145-151, DOI 10.1016/j.topol.2009.04.039. MR.2556089

[14] G. Debs, Espaces héréditairement de Baire (French, with English summary), Fund. Math. 129 (1988), no. 3, 199-206. MR.962541

[15] François G. Dorais and Carl Mummert, Stationary and convergent strategies in Choquet games, Fund. Math. 209 (2010), no. 1, 59-79, DOI 10.4064/fm209-1-5. MR2652592

[16] Edward G. Effros, Convergence of closed subsets in a topological space, Proc. Amer. Math. Soc. 16 (1965), 929-931. MR 0181983

[17] Ryszard Engelking, General topology, 2nd ed., Sigma Series in Pure Mathematics, vol. 6, Heldermann Verlag, Berlin, 1989. Translated from the Polish by the author. MR 1039321

[18] R. C. Haworth and R. A. McCoy, Baire spaces, Dissertationes Math. (Rozprawy Mat.) 141 (1977), 73 pp. MR0431104

[19] Alexander S. Kechris, Classical descriptive set theory, Graduate Texts in Mathematics, vol. 156, Springer-Verlag, New York, 1995. MR.1321597

[20] Erwin Klein and Anthony C. Thompson, Theory of correspondences, Including applications to mathematical economics, Canadian Mathematical Society Series of Monographs and Advanced Texts, John Wiley \& Sons, Inc., New York, 1984. MR752692

[21] K. Kuratowski, Topology. Vol. I, New edition, revised and augmented. Translated from the French by J. Jaworowski, Academic Press, New York-London; Państwowe Wydawnictwo Naukowe, Warsaw, 1966. MR0217751

[22] Keye Martin, Topological games in domain theory, Topology Appl. 129 (2003), no. 2, 177186, DOI 10.1016/S0166-8641(02)00147-5. MR.1961398

[23] John C. Oxtoby, The Banach-Mazur game and Banach category theorem, Contributions to the theory of games, vol. 3, Annals of Mathematics Studies, no. 39, Princeton University Press, Princeton, N. J., 1957, pp. 159-163. MR0093741

[24] E. Porada, Jeu de Choquet (French), Colloq. Math. 42 (1979), 345-353. MR.567573

[25] Rastislav Telgársky, Remarks on a game of Choquet, Colloq. Math. 51 (1987), 365-372. MR.891306

[26] H. E. White Jr., Topological spaces that are $\alpha$-favorable for a player with perfect information, Proc. Amer. Math. Soc. 50 (1975), 477-482. MR0367941

[27] László Zsilinszky, Polishness of the Wijsman topology revisited, Proc. Amer. Math. Soc. 126 (1998), no. 12, 3763-3765, DOI 10.1090/S0002-9939-98-04526-2. MR1458275

[28] László Zsilinszky, On $\beta$-favorability of the strong Choquet game, Colloq. Math. 125 (2011), no. 2, 233-243, DOI 10.4064/cm125-2-8. MR2871316

Department of Mathematics and Computer Science, The University of North Carolina at Pembroke, Pembroke, North Carolina 28372

E-mail address: laszlo@uncp.edu 